\title{
Effects of different shear rates on the attachment and detachment of platelet thrombi
}

\author{
XIAOFENG SHI ${ }^{*}$, JICHUN YANG ${ }^{*}$, JIANSONG HUANG ${ }^{*}$, ZHANGBIAO LONG, \\ ZHENG RUAN, BING XIAO and XIAODONG XI
}

\begin{abstract}
Department of Hematology, Shanghai Institute of Hematology, State Key Laboratory of Medical Genomics, Collaborative Innovation Center of Hematology, Sino-French Research Center for Life Sciences and Genomics, Ruijin Hospital, School of Medicine, Shanghai Jiao Tong University, Shanghai 200025, P.R. China
\end{abstract}

Received March 19, 2015; Accepted January 15, 2016

DOI: $10.3892 / \mathrm{mmr} .2016 .4825$

\begin{abstract}
Thrombosis and hemostasis take place in flowing blood, which generates shear forces. The effect of different shear rates, particularly pathological forces, on platelet thrombus formation remains to be fully elucidated. The present study observed the morphological characteristics and hierarchical structure of thrombi on the collagen surface at a wide range of wall shear rates (WSRs) and examined the underlying mechanisms. Calcein AM-labeled whole blood was perfused over a collagen-coated surface at different shear rates set by a Bioflux 200 microfluidic device and the thrombi formed were assessed for area coverage, the height and the hierarchical structure defined by the extent of platelet activation and packing density. The factors that affect thrombus formation were also investigated. Platelet thrombus formation varied under different WSRs, for example, dispersed platelet adhesion mixed with erythrocytes was observed at $125-250 \mathrm{~s}^{-1}$, extensive and thin platelet thrombi were observed at $500-1,500 \mathrm{~s}^{-1}$, and sporadic, thick thrombi were observed at pathological WSRs of 2,500-5,000 s-1 which showed a tendency to be shed. With increasing WSRs, the height of the thrombi showed an increasing linear trend, whereas the
\end{abstract}

Correspondence to: Professor Xiaodong Xi, Department of Hematology, Shanghai Institute of Hematology, State Key Laboratory of Medical Genomics, Collaborative Innovation Center of Hematology, Sino-French Research Center for Life Sciences and Genomics, Ruijin Hospital, School of Medicine, Shanghai Jiao Tong University, 197 Second Ruijin Road, Shanghai 200025, P.R. China E-mail: xi_xiaodong1955@163.com

*Contributed equally

Abbreviations: WSR, wall shear rate; vWF, von Willebrand factor; 3D, three-dimensional; PPACK, D-phenylalanyl-L-prolyl-L-arginine chloromethyl ketone dihydrochloride; GP, glycoprotein; EDTA, ethylenediamine tetraacetic acid; PBS, phosphate-buffered saline

Key words: shear rates, platelet thrombus formation, under flow, balance, collagen total fluorescence intensity and area of the thrombi exhibited a parabolic curve-like change, with a turning point at a WSR of $2,500 \mathrm{~s}^{-1}$. The number of thrombi, the average fluorescence intensity and the area per thrombus showed similar trends, with an initial upwards incline followed by a decline. The thrombi formed at higher WSRs had a thicker shell, which led to a more densely packed core. Platelet thrombus formation under shear-flow was regulated by the adhesive strength, which was mediated by receptor-ligand interaction, the platelet deposition induced by shear rates and the detachment by the dynamic force of flow. This resulted in a balance between thrombus attachment, including adhesion and aggregation, and detachment. Collectively, compared with physiological low WSRs, pathological high WSRs caused thicker and more easily shed thrombi with more condensed cores, which was regulated by an attachment-detachment balance. These results provide novel insights into the properties of thrombus formation on collagen at different WSRs, and offers possible explanations for certain clinical physiopathological phenomena, including physical hemostasis and pathological thrombosis.

\section{Introduction}

Platelet adhesion and aggregation at sites of vascular injury contribute to the arrest of bleeding, but also to the occlusion of atherosclerotic arteries causing cardiovascular and cerebrovascular diseases $(1,2)$. These events take place in flowing blood, which generates shear forces. Typical ranges of wall shear rates (WSRs) in vivo are as follows: 20-200 s-1 for veins, $300-800 \mathrm{~s}^{-1}$ for large arteries, $500-1,600 \mathrm{~s}^{-1}$ for arterioles and $800-10,000 \mathrm{~s}^{-1}$ for atherosclerotic stenosed arteries (3). Various fluidic devices have been designed to mimic the hemodynamic conditions of the vasculature $(4,5)$ and artificial surfaces coated with different substrates, including collagen, fibrinogen, von Willebrand factor (vWF) and laminin, have been used to simulate the subendothelial matrices (6-8). Among these, collagen fibrils are the most abundant component of the extracellular matrix (9) and considered to be essential for platelet thrombus formation following vessel wall injury (10). Collagen-coated surfaces bear the closest resemblance to physiopathological conditions in vivo (6) and are, thus preferred for flow assays, according to the recommendation by the Biorheology 
Subcommittee of the SSC of the ISTH (11). Although the characteristics of thrombus formation over collagen surfaces at different WSRs have been reported previously, these observations are either limited in relatively lower physiological ranges $(6,12-16)$, or lacking detailed description (17). The precise characteristics of thrombus formation at high pathological WSRs remain to be fully elucidated. Previously, platelet activation parameters, including fibrinogen binding $(\alpha \operatorname{IIb} \beta 3$ activation) and the expression of P-selectin (platelet $\alpha$-granule secretion) were used to evaluate the levels of activation $(18,19)$ and regional architecture of the thrombi (20-23). However, the hierarchical structure of thrombi formed at different WSRs, particularly pathological WSRs, remains to be elucidated.

Novel microfluidic devices $(24,25)$ with dimensions of micrometers can provide higher WSRs with a reasonable whole blood volume $(0.1-1 \mathrm{ml})$ requirement, which makes comparative investigations among a wider range of WSRs possible. Previous observations on thrombus formation in whole blood under flow were based on either the fluorescence labels $(6,7,14,15)$ or washing off erythrocytes following stopping of flowing (26). Reports involving the direct real-time visualization of the thrombotic process on collagen under different WSRs using optical microscopy have been limited to only a few groups (27-29). In the present study, using the microfluidic Bioflux 200 device, high-resolution video microscopy and confocal three-dimensional (3D) imaging, the characteristics of platelet thrombi formed at pathological WSRs were examined and compared with physiological WSRs, and the underlying mechanisms were investigated.

\section{Materials and methods}

Antibodies and reagents. Mouse anti-human monoclonal SZ-2 antibody specific for integrin glycoprotein (GP)Ib was provided by Professor Changgeng Ruan (Jiangsu Institute of Hematology, Suzhou, China) and murine anti-human integrin $\alpha \operatorname{IIb} \beta 3$ monoclonal antibody 7E3 was provided by Dr Junlin Liu (Shanghai Jiao Tong University School of Medicine, Shanghai, China). Monoclonal mouse anti-human integrin $\alpha 2 \beta 1$ blocking antibody (Gi9) was purchased from Acris Antibodies GmbH (Herford, Germany; cat. no. BM2545). Fluorescein isothiocyanate (FITC)-labeled mouse anti-human CD62 P (P-selectin) monoclonal antibody was purchased from Beckman Coulter (Fullerton, CA, USA; cat. no. A07790). Polyclonal rabbit anti-human fibrinogen antibody was purchased from Dako-An (Agilent Technologies, Inc., Santa Clara, CA, USA; cat. no. A0080) and Alexa fluor 488 affinipure donkey anti-rabbit IgG (heavy+light) was purchased from Jackson ImmunoResearch Laboratories, Inc. (West Grove, PA, USA; cat. no. 711-547-003). Multimer purified human vWF was purchased from Hematologic Technologies Inc. (Essex Junction, VT, USA; cat. no. HCVWF-0190). Calcein AM was purchased from Invitrogen (Thermo Fisher Scientific, Inc., Waltham, MA, USA; cat. no. C1430). Equine type I fibrillar collagen was purchased from Chrono-log Corporation (Haverton, PA, USA; cat. no. 385). D-phenylalanyl-L-prolyl-L-arginine chloromethyl ketone dihydrochloride (PPACK) was purchased from Enzo Life Sciences, Inc. (Farmingdale, NY, USA; cat. no. BML-P1117-0025). All other biochemical reagents were obtained from Sigma-Aldrich (St. Louis, MO, USA).
Perfusion assays. Perfusion assays were performed, according to a modified method of Conant et al (30). Briefly, microfluidic channels with cross-sectional dimensions of $250 \mu \mathrm{m}$ in width and $75 \mu \mathrm{m}$ in height (Bioflux 200; Labtech; Fluxion Biosciences, Inc., South San Francisco, CA, USA) were coated with type I collagen $(20 \mu \mathrm{g} / \mathrm{ml}$ dissolved in $0.02 \mathrm{M}$ acetic acid) or vWF $(10 \mu \mathrm{g} / \mathrm{ml})$. As collagen with concentrations $>40 \mu \mathrm{g} / \mathrm{ml}$ occludes microfluidic channels, a concentration of $20 \mu \mathrm{g} / \mathrm{ml}$ was used. A total of 3-8 $\mathrm{ml}$ whole blood was intravenously collected into PPACK $(150 \mu \mathrm{M})$ from healthy volunteers, including 10 males and 10 females aged between 18 and 35 years old, who had provided informed consent, with the approval of the Human Research Ethics Committee of Ruijin Hospital, Shanghai Jiaotong University School of Medicine (Shanghai, China). The experiments were performed in the $2 \mathrm{~h}$ following blood collection. In a quantitative assay, the fluorescent dye, calcein AM $(4 \mu \mathrm{M})$, was used to label the platelets. For the inhibition assays of the antagonists, SZ-2 $(5 \mu \mathrm{g} / \mathrm{ml})$, Gi9 $(50 \mu \mathrm{g} / \mathrm{ml}), 7 \mathrm{E} 3(10 \mu \mathrm{g} / \mathrm{ml})$, or the disodium salt of ethylenediamine tetraacetic acid (EDTA; $5 \mathrm{mM}$ ), were added to the blood. The different WSRs were set using the Bioflux 200 software. The whole blood was perfused through the microfluidic channels at predefined WSRs $(125,250,500,1,000,1,500$, 2,500, 3,750 and 5,000 s-1) for 4-5 min. To perfuse at 5,000 s for $5 \mathrm{~min}, 500 \mu \mathrm{l}$ whole blood was required, which was the maximal volume that the inlet was able to hold. Thrombus formation was recorded using an inverted fluorescence microscope and charged-couple device camera (Nikon eclipse Ti-S; Nikon Corporation, Tokyo, Japan). A video was recorded in real-time at a rate of 100 frames per second. The total fluorescence intensity, total area coverage and mean fluorescence intensity per visual field were calculated automatically by the Bioflux 200 software with a threshold of 15 , at which point the single platelet was visible, but the background was not.

$3 D$ imaging assay. Following perfusion of the calcein AM-labeled whole blood for $4.5 \mathrm{~min}$ at different WSRs, the microfluidic channels were washed using phosphate-buffered saline (PBS) at $125 \mathrm{~s}^{-1}$ for $5 \mathrm{~min}$, following which the samples were examined and scanned using a Leica laser confocal microscope (Leica TCS SP8; Leica Microsystems, Wetzlar, Germany). 3D images were created through the stacking of a collection of two-dimensional images, with deconvolution to eliminate out-of-focus information, using LAS AF Lite software (2.6.0 build 7266; Leica Microsystems). Z-stack scanning was performed at intervals of $0.3 \mu \mathrm{m}$ with a $60 \mathrm{X}$ objective. The height of every thrombus was calculated using LAS X 3D analysis software (1.1; Leica Microsystems).

Platelet tracking assay. The PPACK-anticoagulated blood was perfused through a vWF-coated $(10 \mu \mathrm{g} / \mathrm{ml})$ surface at different WSRs. Subsequently, the platelet adhesion on vWF was recorded in real-time using an inverted fluorescence microscope and charged-couple device camera (Nikon eclipse Ti-S; Nikon Corporation). A total of 10 consecutive frames were captured every $0.54 \mathrm{sec}$ following the onset of blood flow, which were overlapped to show the platelet tracking path lines and analyzed for mean velocity of platelet movement using Bioflux 200 software. 
A
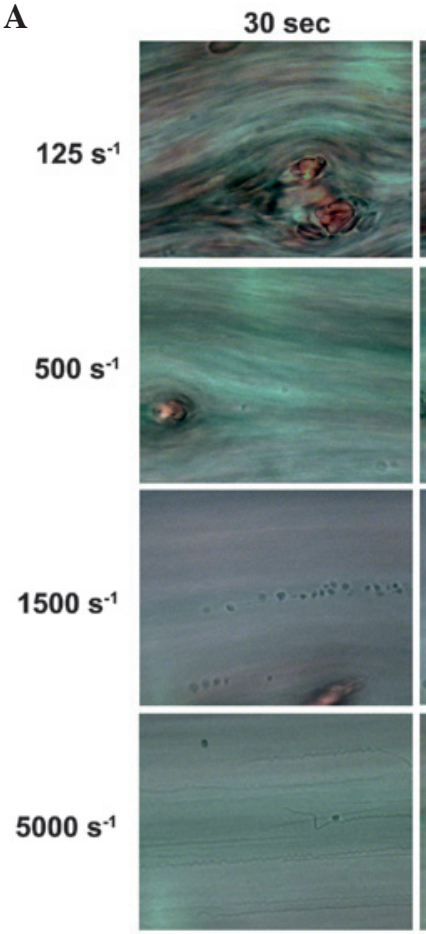
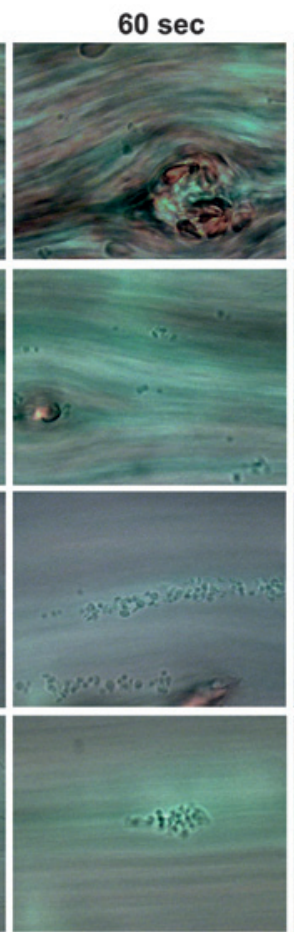

B
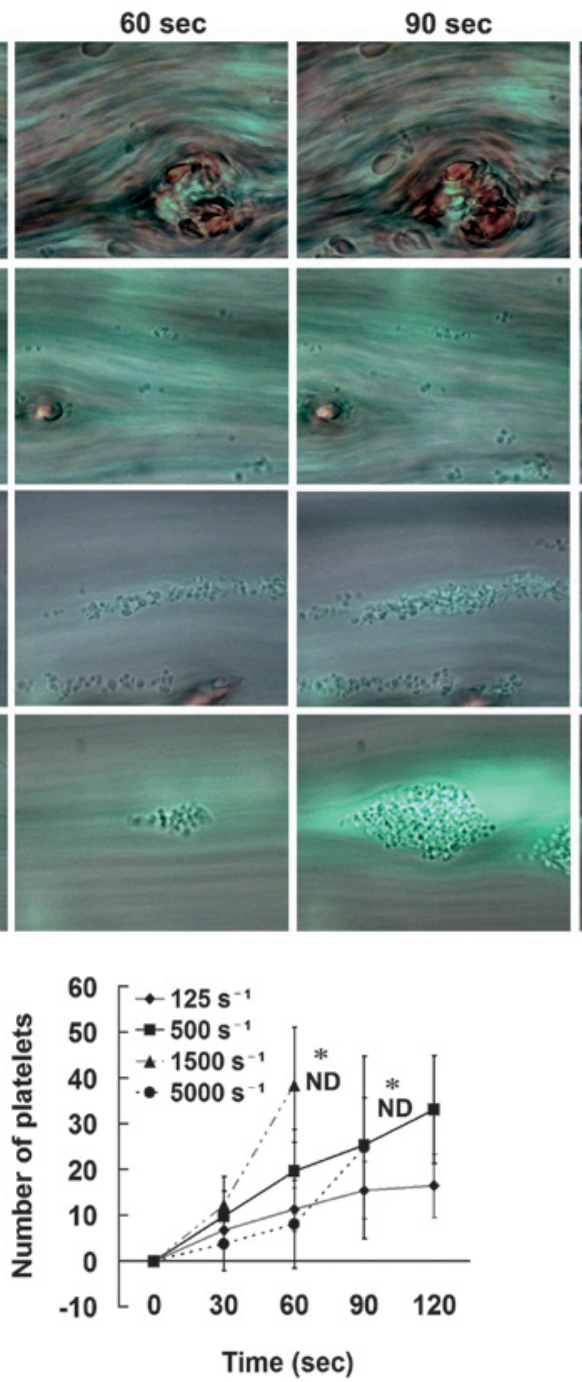

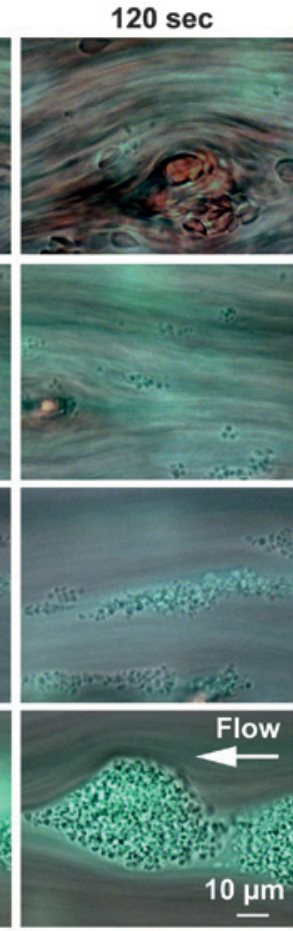

$10 \underline{\mu m}$

Figure 1. Characteristics of thrombus formation on collagen at different WSRs. (A) Thrombus formation at the indicated time points. The images at WSRs of 500 and $125 \mathrm{~s}^{-1}$ are unclear due to low flow velocity-induced artifact. (B) Number of adhesive platelets. Data are presented as the mean \pm standard deviation of four separate experiments from three donors. *ND, quantification not performed as platelets overlapped. The thrombi formed were dispersed and mixed with erythrocytes at $125 \mathrm{~s}^{-1}$, extensive and thin at $500 \mathrm{~s}^{-1}$, densely covered at 1,500 s $\mathrm{s}^{-1}$, but sporadic and thick at 5,000 $\mathrm{s}^{-1}$. During the first $120 \mathrm{~seconds}$, the thrombus growth rates gradually increased with the elevation of WSR. WSR, wall shear rate.

Definition of the hierarchical structure of thrombi by the extent of platelet activation and packing density. Defining the hierarchical structure of the thrombi was performed, according to previously described methods $(21,22)$. Following perfusion of the PPACK-anticoagulated whole blood, without the addition of calcein AM, for 4.5 min at different WSRs, the microfluidic channels were washed using PBS at $125 \mathrm{~s}^{-1}$ for $1 \mathrm{~min}$. Then thrombi were then stained by perfusion at $125 \mathrm{~s}^{-1}$ for 2 min with FITC-labeled anti-P-selectin monoclonal antibody (1:100) or polyclonal rabbit anti-human fibrinogen antibody (1:500), followed by incubation at room temperature for 30 min with Alexa fluor 488-labeled donkey anti-rabbit secondary antibody (1:500), all in PBS with calcium and magnesium. Following $30 \mathrm{~min}$ of static staining at room temperature, unbound labeling was washed off using PBS at $125 \mathrm{~s}^{-1}$. The total thrombus area was defined by the thrombus outline in brightfield; the area of the core region was defined by the fibrinogen-binding fluorescence using an inverted fluorescence microscope (Nikon eclipse Ti-S; Nikon Corporation). Subtracting the fibrinogen-binding area from the total thrombus area defined the area of the outer shell region. The marked fibrinogen-binding borderline between the core and shell was defined as the boundary zone. The area and the mean fluorescence intensity of the distinct regions were measured by gating the images with NIS Elements imaging analysis software (version NIS Elements D; Nikon Corporation).

Statistical analysis. The SPSS 18 statistical software program was used for statistical analysis. Quantitative data are expressed as the mean \pm standard deviation. Differences between groups were assessed using Student's t-test. $\mathrm{P}<0.05$ was considered to indicate a statistically significant difference.

\section{Results}

Morphological characteristics and hierarchical structure of thrombi formed at different WSRs. It is known that the WSR is $\sim 125 \mathrm{~s}^{-1}$ in veins, ranges between 450 and $500 \mathrm{~s}^{-1}$ in medium-sized arteries, including coronary arteries $(3,31,32)$, is $\sim 1,500 \mathrm{~s}^{-1}$ in arterioles (3) and reaches a pathological level 

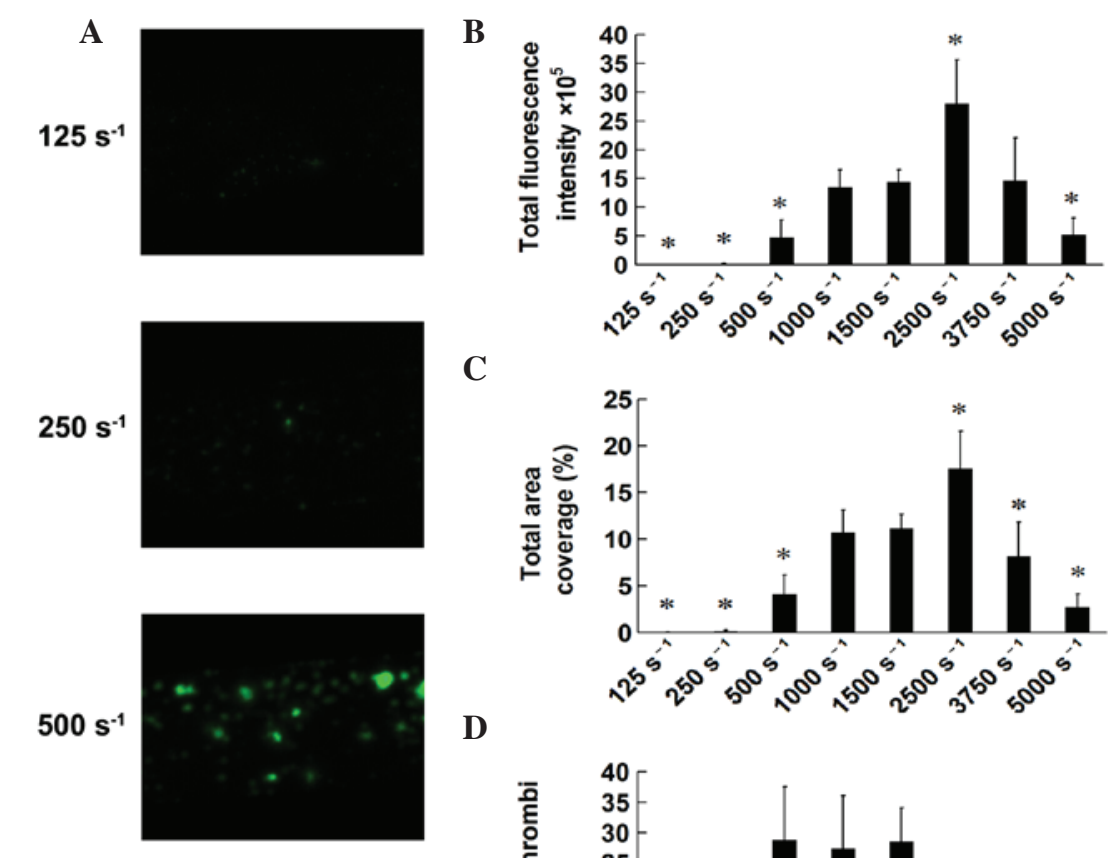

C
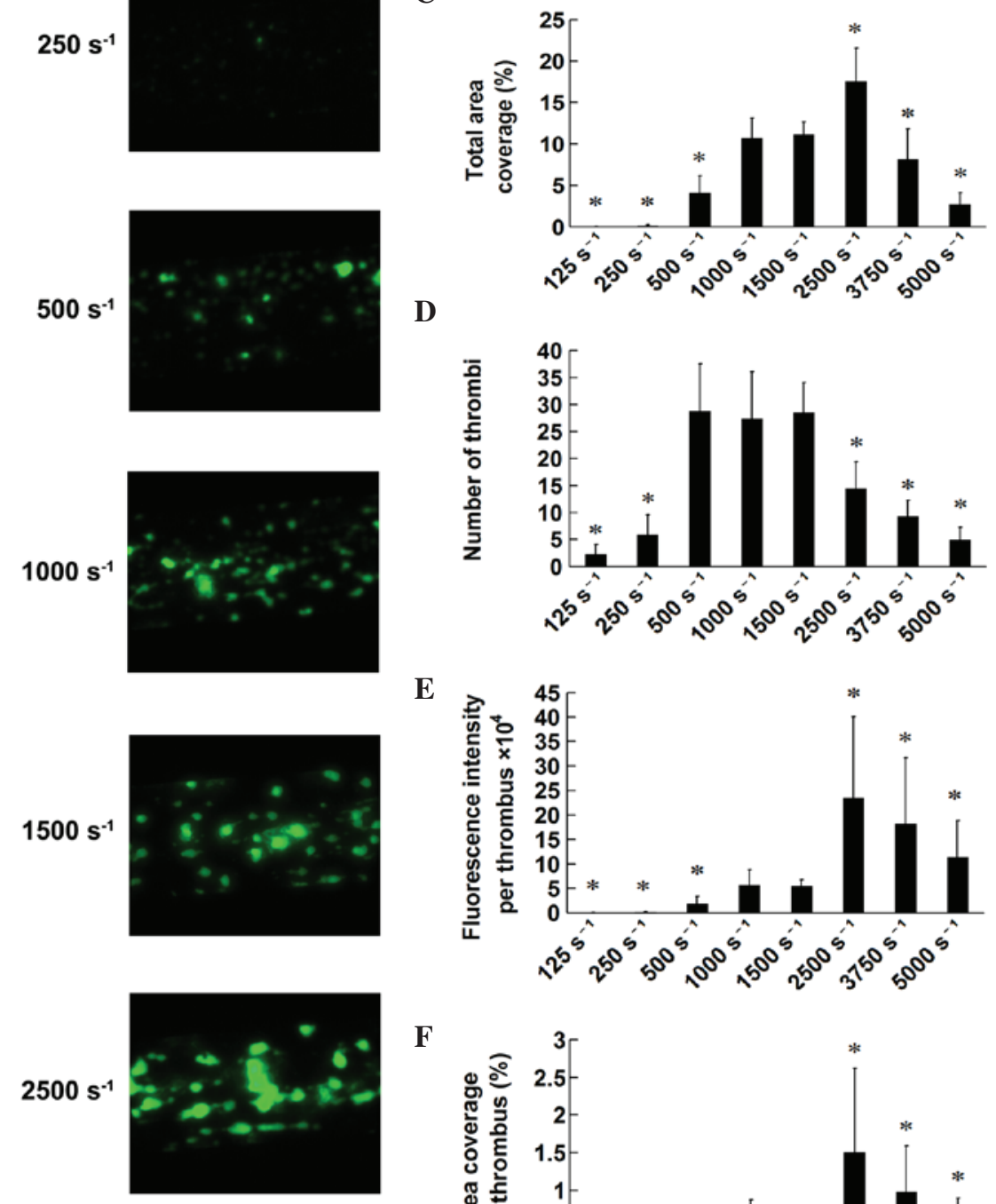

E
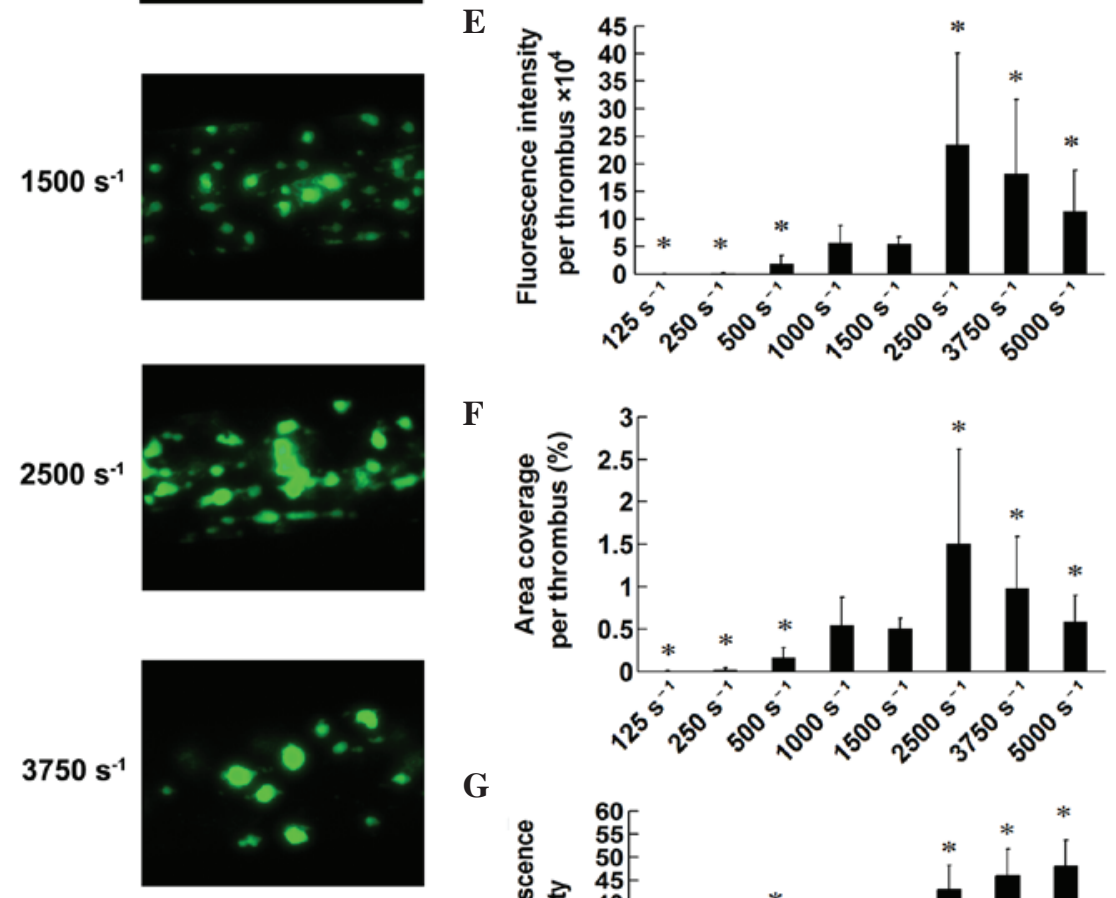

$\mathbf{F}$

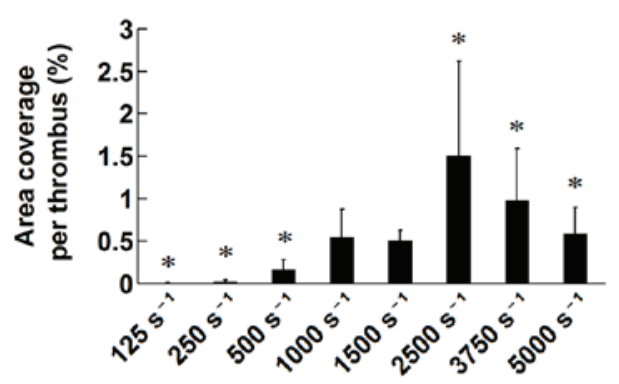

G
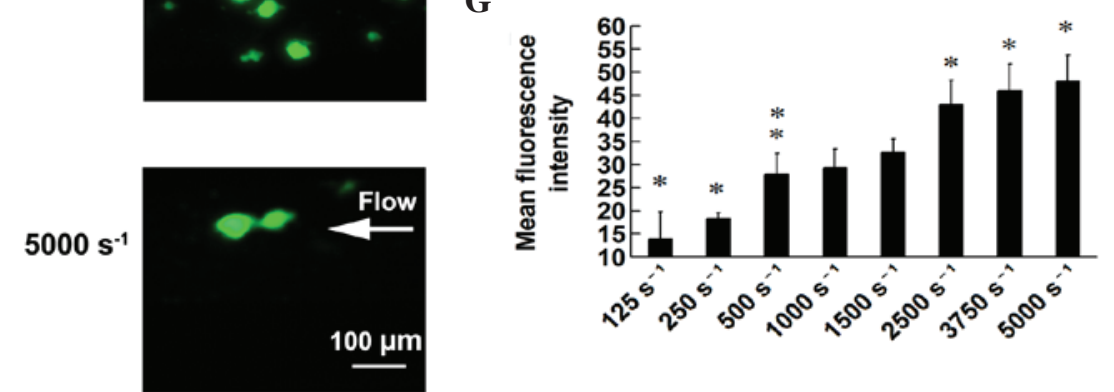

Figure 2. Quantitative analysis of thrombus formation at different WSRs. Calcein AM-labeled whole blood was perfused over a collagen-coated surface at indicated WSRs for 4.5 min. (A) Representative images. (B) Total fluorescence intensity. (C) Total area coverage. (D) Number of thrombi. (E) Fluorescence intensity per thrombus. (F) Surface area coverage per thrombus. (G) Mean fluorescence intensity (total fluorescence intensity/total area coverage). ${ }^{*}<0.01$ and ${ }^{*} \mathrm{P}<0.05$, compared with that at $1500 \mathrm{~s}^{-1}$. Results are presented as the mean \pm standard deviation from at least 40 randomly-selected visual fields of at least four independent experiments from three donors. WSR, wall shear rate. 
A

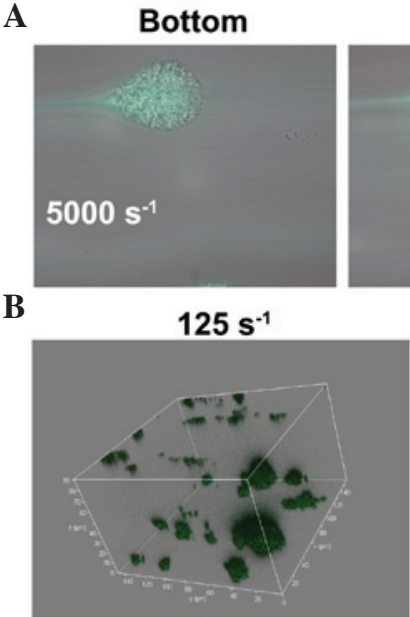

$2500 \mathrm{~s}^{-1}$

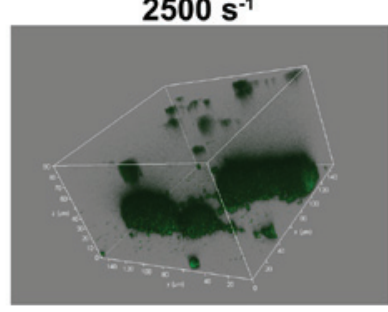

Middle

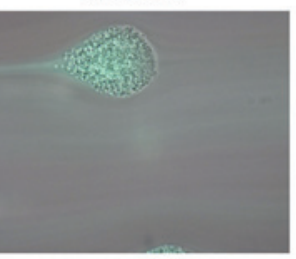

$500 \mathrm{~s}^{-1}$

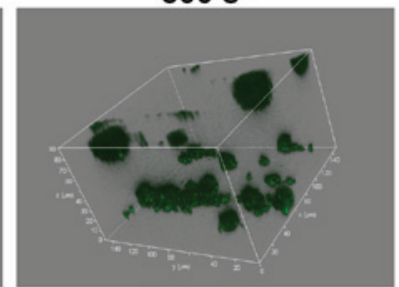

$5000 \mathrm{~s}^{-1}$

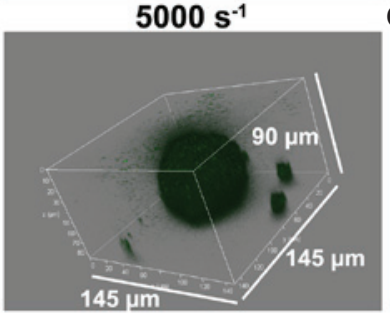

Upper
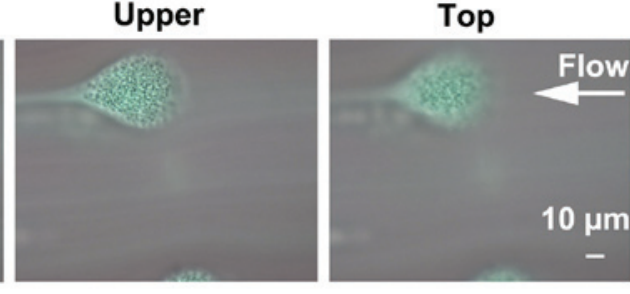

$1500 \mathrm{~s}^{-1}$
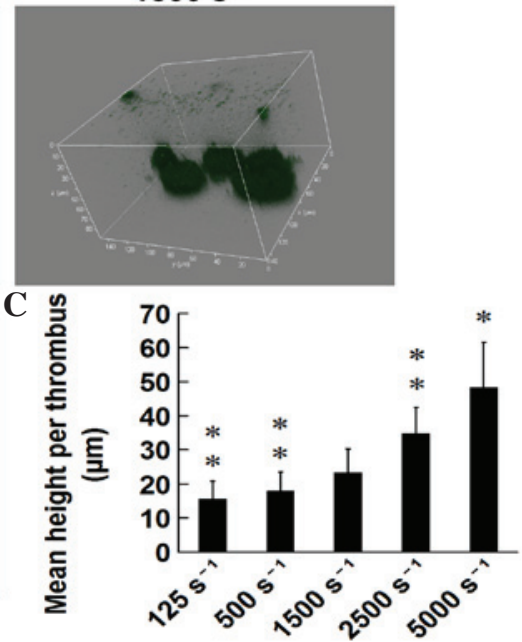

Figure 3. Elevated WSRs cause thicker thrombi. (A) A large, thick thrombus was formed following perfusion for 1.5 min at $5,000 \mathrm{~s}^{-1}$, the bottom, middle, upper and top parts of which are shown. (B) Three-dimensional imaging of thrombi at indicated WSRs. (C) Calculated height per thrombus. ${ }^{*} \mathrm{P}<0.01$ and ${ }^{* *} \mathrm{P}<0.05$, compared with that at $1500 \mathrm{~s}^{-1}$. Results are presented as the mean \pm standard deviation from 12-25 randomly-selected thrombi from three experiments for every WSR examined. WSR, wall shear rate.

between 1,500 and $10,000 \mathrm{~s}^{-1}$ close to the stenotic apex in atherosclerotic arteries $(3,33)$. In the present study, the above WSRs were selected, and the process of thrombus formation was observed under optical microscopy in real-time. In this system ex vivo, the thrombus resembled a platelet thrombus, as PPACK-anticoagulated blood was used, which did not allow thrombin generation or fribrin formation. The number of adhered and aggregated platelets increased in a time-dependent manner at all WSRs in the first 2 mins (Fig. 1). At a low WSR of $125 \mathrm{~s}^{-1}$, platelet adhesion was shown in only a thin layer, and the thrombi were small and mixed with relatively larger-volume erythrocytes. At a WSR of 1,500 s $\mathrm{s}^{-1}$, the platelets gradually formed disseminated and thick funicular thrombi, which were elongated in the direction of flow. The characteristics of platelet adhesion and aggregation were observed at a WSR of $500 \mathrm{~s}^{-1}$ between 1,500 and $125 \mathrm{~s}^{-1}$. At a WSR of 5,000 s $\mathrm{s}^{-1}$, initial adhesion of the platelets was limited and unstable, however, once the first platelet adhered irreversibly to the collagen surface, the thrombus grew rapidly. At 5,000 s $\mathrm{s}^{-1}$, the large and thick thrombus exhibited a tadpole-like appearance, with the head against, and the tail in the direction of flow (Fig. 1). To accurately quantify the number of thrombi, the platelets were stained using calcein AM, which has no effect on the function of platelets (30). The total fluorescence intensity and total area coverage generated a parabolic curve-like change with a peak at 2,500 s-1 (Fig. 2A-C). The number of thrombi were low at the lower WSRs of 125 and $250 \mathrm{~s}^{-1}$, and increased markedly, reaching a plateau at moderate WSRs of 500, 1,000 and $1,500 \mathrm{~s}^{-1}$, followed by a gradual decrease at high WSRs of 2,500, 3,750 and 5,000 s ${ }^{-1}$ (Fig. 2D). The fluorescence intensity and area coverage per thrombus were low at the lower WSRs of 125 and $250 \mathrm{~s}^{-1}$, increased at the moderate WSRs of 500, 1,000 and 1,500 $\mathrm{s}^{-1}$, and were most marked at the high WSR of $2,500 \mathrm{~s}^{-1}$. The intensity gradually decreased at higher WSRs between 2,500 and 5,000 s-1 although remained higher than that at moderate WSRs (Fig. 2E and F). The mean fluorescence intensity, roughly reflecting the height of the thrombi, increased progressively with WSR increase, and reached a maximum at 5,000 $\mathrm{s}^{-1}$ (Fig. 2G). At 5,000 s $\mathrm{s}^{-1}$, the bottom, middle, upper and top regions of the thrombi were observed through modulation of the focal length of the microscope, with an estimated height of 40-60 $\mu \mathrm{m}$ (Fig. 3A). The 3D images showed that the mean heights of the thrombi showed a shear-dependent increase between 16.8 and $48.7 \mu \mathrm{m}$. (Fig. 3B and C). The overlapping of multiple thrombi was also observed at 2,500 s-1, which appeared to enlarge the area coverage of the individual thrombus, but did not affect its height (Fig. 3B), resulting in the peak of area coverage per thrombus at $2,500 \mathrm{~s}^{-1}$. Due to the cessation of flow, the thrombi at $5,000 \mathrm{~s}^{-1}$ acquired a mound-like appearance, rather than a tadpole-like appearance (Fig. 3B). It was also shown that the thrombi were located at the bottom, as well as the top of the channel, possibly due to the collagen coating surrounding the channel (Fig. 3B).

The activation status of the thrombi was stratified, based on fibrinogen-binding fluorescence intensity, which was observed as a lightly stained inner core, deep staining of the surrounding boundary zone and a pale shell outside (Fig. 4A and $\mathrm{B}$ ). The low fluorescence intensity in the core region reflects a reduction in porosity and an increase in the packing 


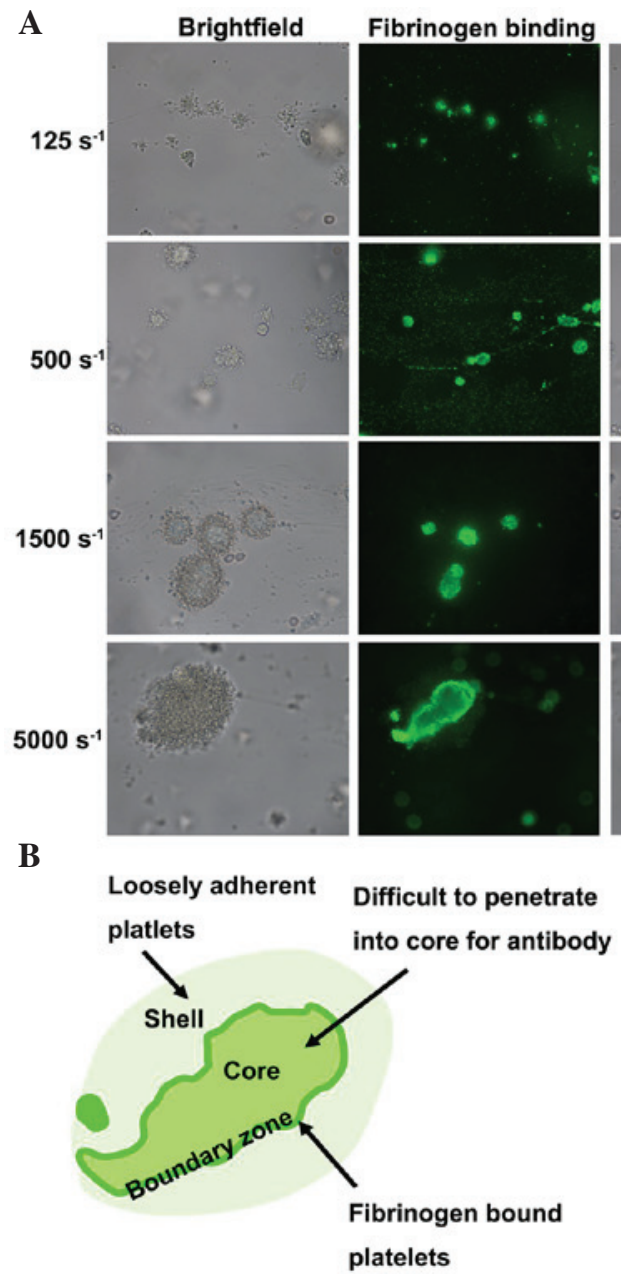

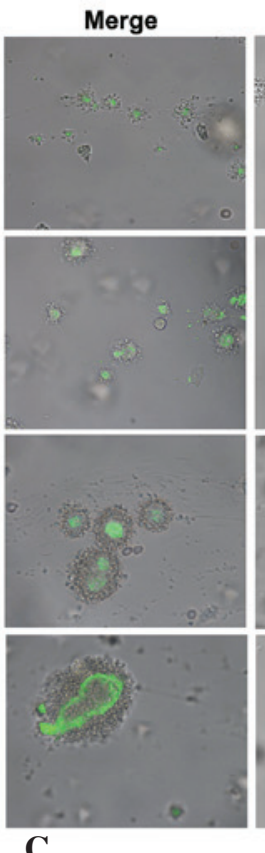

C

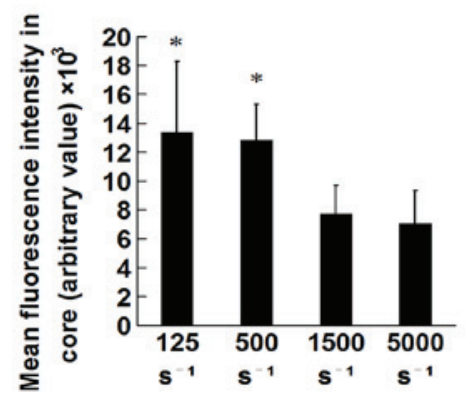

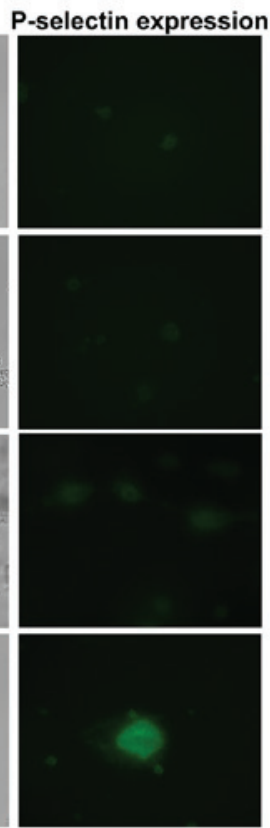

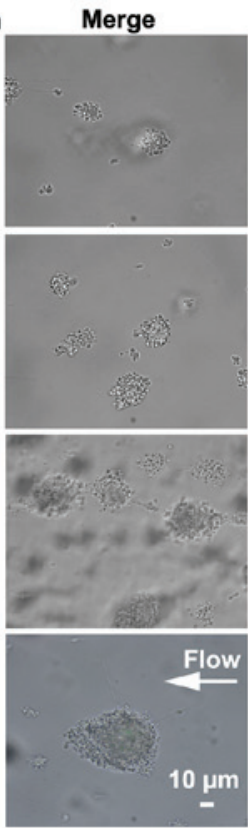

D

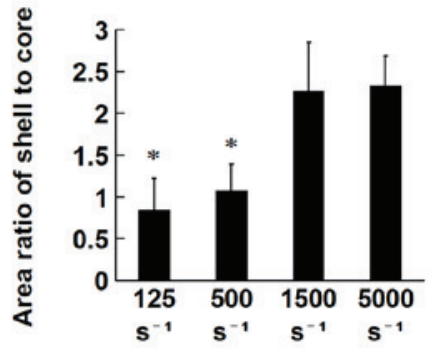

Figure 4. Thrombi formed at high WSRs have higher shell-core rates and more closely packed cores. Whole blood, without the addition of calcein AM, was perfused over a collagen-coated surface for $4.5 \mathrm{~min}$ at the indicated WSRs. The thrombi were stained to evaluate the hierarchical structure, based on the extent of platelet activation and packing density. anti-fibrinogen antibody was used to assess fibrinogen binding and P-selectin antibody was used to determine the expression of P-selectin. (A) Representative images under brightfield and fluorescence microscopy, and of the two merges. (B) Schematic diagram of merged images of fibrinogen binding showing the stratified thrombus structure with core, boundary zone and shell. (C) Mean fluorescence intensity in the core at the indicated WSRs. (D) Area ratio of shell:core at indicated WSRs. ${ }^{*} \mathrm{P}<0.01$ and ${ }^{* *} \mathrm{P}<0.05$, compared with that at $1,500 \mathrm{~s}^{-1}$. Results are presented as the mean \pm standard deviation from 20-25 randomly-selected thrombi from three experiments for every WSR examined. WSR, wall shear rate.

density of the core (22). Therefore, the present study analyzed the mean fluorescence intensity in the core to estimate the extent of packing density of the core in the thrombi formed at different WSRs. The results showed that the thrombi formed at higher WSRs had lower mean fluorescence intensity in the core, therefore, with increasing WSR, the thrombi had a more closely packed core (Fig. 4C). As it has been reported that the shell provides protection for the core (22), the present study further analyzed the area ratio of shell to core. The results demonstrated that the area ratio of shell:core increased with WSR, indicating that the thrombi formed at higher WSRs had a thicker shell, compared with those formed at low WSRs (Fig. 4D). As the expression of P-selectin on the activated platelet surface is lower than that of the fibrinogen receptor, $\alpha \operatorname{IIb} \beta 3$ (34), the fluorescence intensity of the former was weak, compared with that of the latter, which made analysis difficult (Fig. 4A).

Underlying mechanisms for thrombus formation at different WSRs. The nature of the receptors involved in adhesion and aggregation at different WSRs was probed using function-inhibiting monoclonal antibodies against-GPIb (SZ-2), $\alpha 2 \beta 1$ (Gi9) or $\alpha \mathrm{IIb} \beta 3$ (7E3). Potent divalent cation chelating agents (EDTA) served as a positive control. The inhibition of GPIb by SZ-2 completely eradicated the initial adhesion of platelets at moderate-high WSRs of 1,500 and $5,000 \mathrm{~s}^{-1}$. Inhibition of $\alpha 2 \beta 1$ by Gi9 had significant inhibitory effects on thrombus formation at high WSRs of 1,500 and $5,000 \mathrm{~s}^{-1}$ with modest inhibitory effects at $500 \mathrm{~s}^{-1}$. The inhibition of $\alpha \mathrm{IIb} \beta 3$ by 7E3 had significant inhibitory effects on aggregation at almost all WSRs (Fig. 5A). These adhesive receptors assisted in resisting the high levels of shear stress.

In order to examine the association between platelet deposition rate and shear rates, microfluidic channels were coated with vWF, which can mediate platelet surface translocation, rather than rapid and brief contact mediated by collagen, making the platelet movement visible. The adhesion of platelets to the vWF-coated surface demonstrated the characteristics of a monolayer, but without overlapping. The number of platelets adhered to the vWF surface exhibited shear rate-dependent increase (Fig. 5B), indicating that elevated WSRs caused platelet deposition. 
A

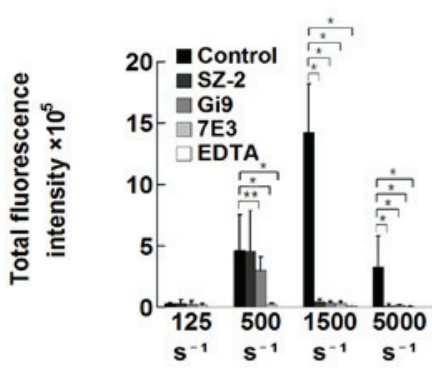

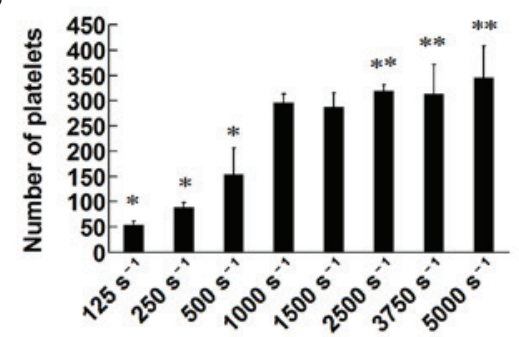

C

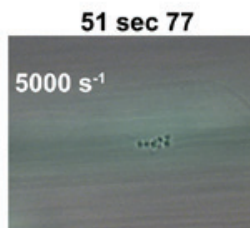

$51 \sec 83$

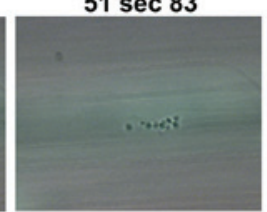

$52 \sec 19$

$52 \sec 25$
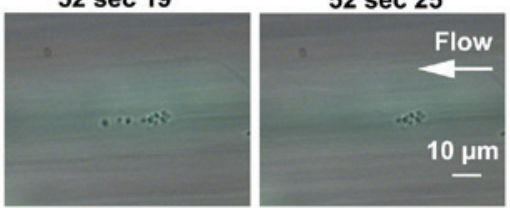

D

$3 \min 1 \mathrm{sec}$

$3 \min 3 \mathrm{sec}$

$3 \min 8 \mathrm{sec}$
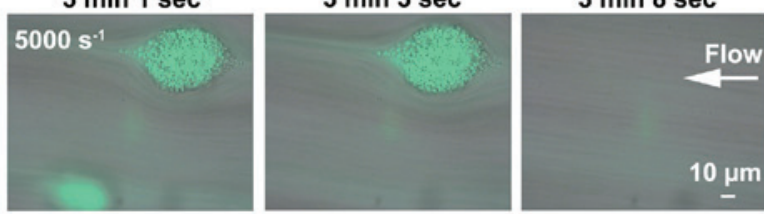

$\mathbf{E}$

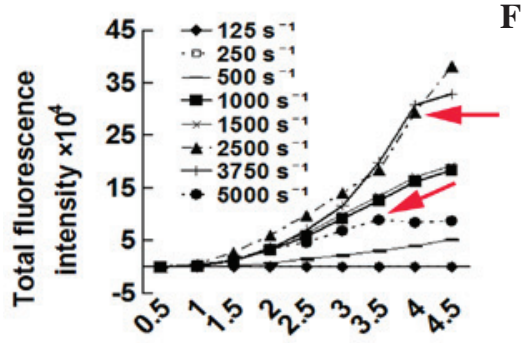

Time (min)

G

$125 \mathrm{~s}^{-1}$

$500 \mathrm{~s}^{-1}$
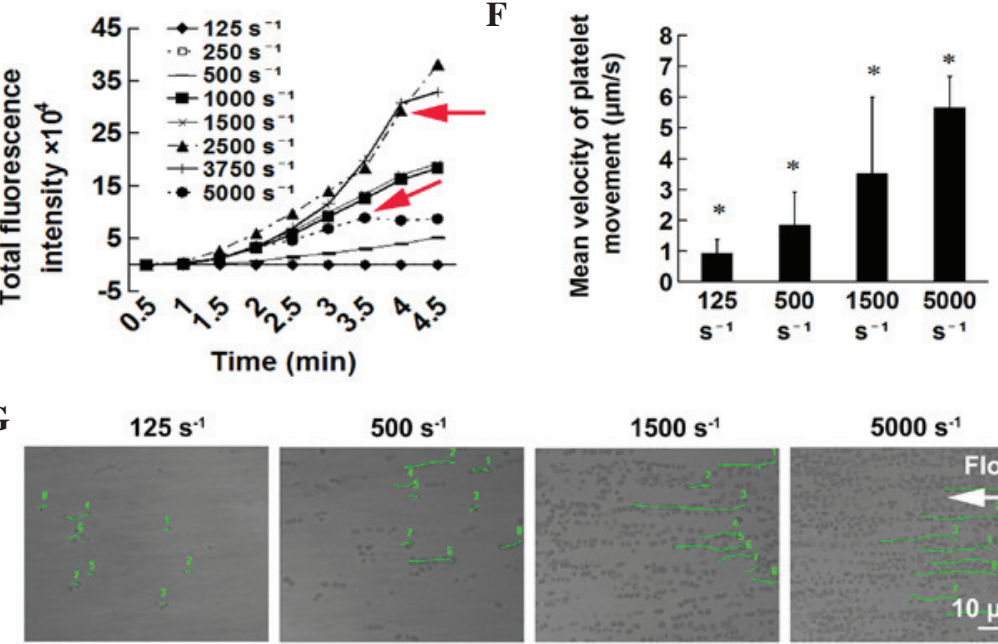

$1500 \mathrm{~s}^{-1}$

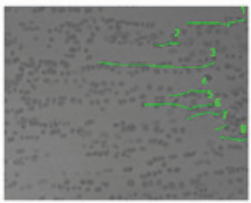

$5000 \mathrm{~s}^{-1}$

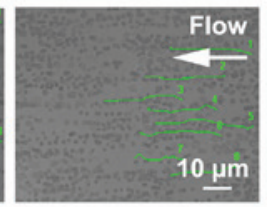

H

Attachment

Detachment

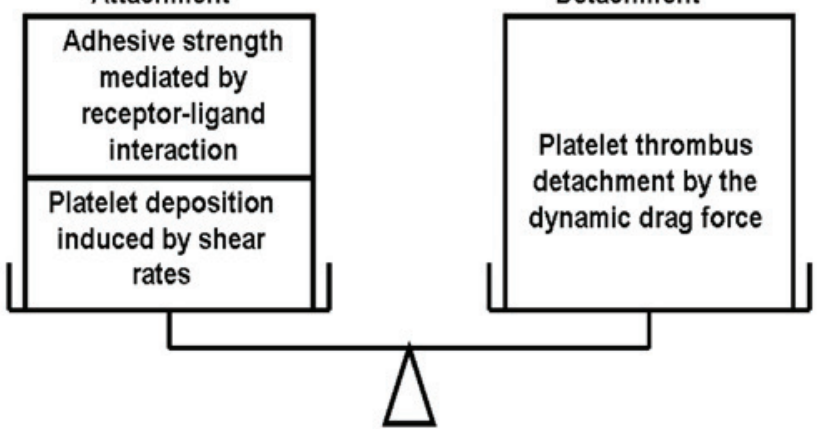

Figure 5. Underlying mechanisms for thrombus formation at different WSRs. (A) Adhesive receptors assist in resisting the drag force imposed by flow. Whole blood was pretreated with no antagonist (control), with calcium chelating agents (EDTA), anti-GPIb (SZ-2), anti- $\alpha 2 \beta 1$ (Gi9) and anti- $\alpha$ IIb $\beta 3$ (7E3) antibodies. Results are presented as the mean \pm standard deviation from 30-45 randomly-selected fields of three independent experiments from three donors. ${ }^{*} \mathrm{P}<0.01$ and ${ }^{* *} \mathrm{P}<0.05$. (B) Elevated WSRs caused platelet deposition. Whole blood was perfused over a von Willebrand factor-coated surface at indicated WSRs. The number of adhesive platelets was counted. Results are presented as the mean \pm standard deviation from at least eight randomly-selected fields of four independent experiments from three donors. (C-G) Elevated WSRs led to shedding of thrombi. " $\mathrm{P}<0.01$ and ${ }^{* * *} \mathrm{P}<0.05$, compared with that at $1,500 \mathrm{~s}^{-1}$. (C) Platelets downstream, tethered to the head of the thrombi, were detached at 5,000 s-1. (D) At 5,000 s ${ }^{-1}$, two thrombi were gradually shed. (E) Fluorescence images were captured every $30 \mathrm{sec}$. In order to reduce the bleaching of fluorescence, a $4 \mathrm{X}$ long-working-distance objective was used, laser intensity was reduced to $1 / 16$ of that mentioned above, and exposure time was extended. The thrombi progressively accumulated with time at all WSRs, with the exception of 5,000 and 3,750 s' , at which the curve exhibited a sharp turn at $\sim 3.5-4 \min$ (red arrows). ${ }^{*} \mathrm{P}<0.01$ and ${ }^{* *} \mathrm{P}<0.05$, compared with that at 1,500 s${ }^{-1}$. The (G) mean velocity of movement of eight randomly-selected platelets was calculated. (F) Platelet tracking path lines of the randomly selected platelets at different WSRs are shown in green lines with assigned numbers. Results are representative of three independent experiments. (H) Schematic diagram of the attachment-detachment balance. WSR, wall shear rate. 
Initial thrombus formation at high WSRs of 5,000 s ${ }^{-1}$ was slower, and the first platelet took $30-60 \mathrm{sec}$ to adhere stably to the surface. The platelets, which were tethered to already irreversibly adhered platelets (the head of thrombi) were washed away (Fig. 5C). When the thrombi grew bigger and thicker for $\sim 2-3$ min following perfusion at high WSRs, they were shed off (Fig. 5D). At WSRs $\leq 2,500 \mathrm{~s}^{-1}$, the thrombi were relatively stable and the curve of total fluorescence intensity showed a time-dependent linear ascent, whereas at high WSRs of 5,000 and $3,750 \mathrm{~s}^{-1}$ the thrombi were shed, causing a sharp turn in the curve at $\sim 3-4 \mathrm{~min}$ (Fig. 5E). To further estimate the dynamic drag force caused by different WSRs, the velocity of platelet translocation on the vWF-coated surface was calculated, which shows high levels of correlation with dynamic drag force imposed by flow (7). The mean velocity of platelet movement elevated gradually with increasing WSR (Fig. 5F and $\mathrm{G})$.

\section{Discussion}

Using high resolution microscopy and a CCD camera, platelet adhesion and aggregation on collagen in flow exhibited distinct structural characteristics. Physiological venous WSR conditions (125-250 s-1) generated uniform, dispersed platelet adhesion and small thrombi; physiological arterial WSRs (500-1,500 $\left.\mathrm{s}^{-1}\right)$ generated extensive and thin platelet thrombi elongated in the direction of flow; whereas elevated pathological WSRs $\left(2,500-5,000 \mathrm{~s}^{-1}\right)$ generated sporadic and thick thrombi. The thrombi consisted of platelets and erythrocytes at low WSRs, and consisted exclusively of platelets at high WSRs, which was in accordance with the fact that red thrombi predominate in veins and white thrombi in arteries in vivo (35). Notably, the high pathological WSR of 5,000 s-1 generated large, thick thrombi with a specific "trochocephalia and slendertail' tadpole-like configuration, and this slendertail disappeared when blood flow stopped. This specific configuration may result from the hemodynamic process, in which the new platelets on the head of the thrombi in the flow upstream rapidly translocated into the low-shear shadow zone downstream, and tethered to the thrombus body gradually. Colace et al (15) described the morphological features of aggregates, however, the shear rate was only between 100 and $2,000 \mathrm{~s}^{-1}$. The observation was fluorescence-dependent, however, it was not directly visible in real-time optical observation and thus certain details were omitted, including erythrocyte aggregates at low WSRs, tadpole-like platelet aggregates at high WSRs and platelet movement.

The fluorescence quantification and 3D imaging indicated that, with increasing WSRs, the height of the thrombi manifested a linear increase, whereas the total fluorescence intensity and area of the thrombi generated a parabolic curve-like change with an inflection point at 2,500 $\mathrm{s}^{-1}$. The number of thrombi, the average fluorescence intensity and the area per thrombus showed a similar trend, with an initial increase followed by a decrease. Previous reports on the effects of WSRs on the height of thrombi are rare, and descriptions of the effects of WSRs on thrombus formation have been limited to relatively low WSRs and varied among groups: Peak platelet accumulation on collagen has been reported at $150 \mathrm{~s}^{-1}$ in a WSR range between 0 and $600 \mathrm{~s}^{-1}$ ) (12), $150 \mathrm{~s}^{-1}$ in a range of $150-1,500 \mathrm{~s}^{-1}$ (14) and
$500 \mathrm{~s}^{-1}$ in a range of $100-1000 \mathrm{~s}^{-1}(15)$, respectively. However, the front part of the parabolic curve-like trendline of the total thrombus formation in the present study was in agreement with the observation of Savage et al (6), which reported that the total thrombi formed on collagen increased gradually at serial WSRs between 100 and 500 to $1,500 \mathrm{~s}^{-1}$. The second part of the parabolic curve-like trendline of the thrombus formation has not been reported previously. Therefore, the present study provided data on higher pathological WSRs.

The thrombi formed under flow had a hierarchical structure defined by the extent of platelet activation and packing density. The inner core was lightly stained, where the platelets were packed and it was difficult for the antibody to penetrate; a colored boundary zone was observed outside the core, where the thrombi grew, and an outer shell was present, where the platelets adhered loosely, similar to the P-selectin-based hierarchical organization of thrombi formed in murine cremaster muscle arterioles following laser injury (20). In the present study, this hierarchical structure of thrombi formed at different WSRs was investigated and it was found that the thrombi formed at higher WSRs had a more closely packed core and thicker shell. The thick shell, which was comprised of recruited platelets with less activity, may protect the platelet-paracrine soluble agonists in the cord, ADP and TXA2, from blood flow 'washout', and these soluble agonists drive platelet full activation, shape change, cohesion and clot retraction $(20,36)$, and eventually lead to a packed core. A higher shell-to-core ratio at higher WSRs indicated a higher degree of protection provided by the shell to the core. However, the thickness of the shell was correlated with the extent of platelet recruitment, which was shear-dependent.

The possible mechanism underlying the above-mentioned shear-based change of thrombus formation was examined in the present study. The results of the function-inhibition assays suggested that the interactions between the GPIb, $\alpha \operatorname{IIb} \beta 3$ and $\alpha 2 \beta 1$ receptors and their ligands contributed to the resistance to the drag force imposed by the flow. The platelet deposition rate gradually increased with elevation of WSR, possibly due to an increase in the volume of blood perfused within a period of time (6) and increased collision frequency of the platelets (37). As a result, the thrombi grew faster at higher WSRs, compared with low WSRs, with the thickest thrombus identified at the highest WSR. This led to the observed linear increase in the height of the thrombi. The present study further examined the dynamic drag force caused by WSRs. As the velocity of platelet movement reflects the dynamic drag force (7), the faster movement of platelets at relatively higher WSRs indicated a more marked dynamic drag force imposed on the platelets. The dynamic drag force was positively correlated with the WSRs and thrombus height, according to the previously described formula $(38,39): R e_{\mathrm{p}}=\gamma H_{\mathrm{p}}^{2} / \mu\left(R e_{\mathrm{p}}\right.$ is the particle Reynolds number, $\gamma$ is the WSR, $H_{\mathrm{p}}$ is the thrombus height and $\mu$ is the blood viscosity). Elevated WSRs or increasing thrombus thickness (depending on the former) significantly increase the likelihood of a detachment event $(17,39)$. Therefore, the higher the WSRs were, the more readily the thrombi were shed, causing a decline in total thrombus formation at WSRs $>2,500 \mathrm{~s}^{-1}$. This explains why the total fluorescence intensity and thrombus area generated a parabolic curve-like change. 
This decreasing trend was not observed in platelet deposition on the vWF-coated surface due to the thin monolayer adhesion.

The adhesive strength, including adhesion to the extracellular matrix and between platelets (mediated by receptor-ligand interaction), platelet deposition induced by WSRs and detachment by dynamic drag force, form a balance in attachment, including adhesion and aggregation, and detachment, or an equilibrium between the forces forming thrombus and those causing shedding (Fig. 5H). The forces produced by shear rates not only pushed the platelets towards the vessel wall to facilitate their deposition, but also pulled them forward in the direction of flow causing shedding. The inflection point of the WSRs, where the condition transforms from facilitating thrombus formation to promoting shedding, was $2,500 \mathrm{~s}^{-1}$.

There are several physiopathological implications of the findings of the present study. The WSR of $500 \mathrm{~s}^{-1}$ represents non-stenosed medium-sized arteries, including the coronary arteries $(3,31,32)$, whereas vasculitis or mechanical effects often result in endothelial injury. The thin and stable thrombi generated by this WSR not only facilitates endothelial repair, but also sustain vascular patency. The WSR of $1,500 \mathrm{~s}^{-1}$ reflects the condition in arterioles where hemostasis primarily develops following trauma (3). The thrombi with increased thickness generated by this WSR are sufficient for the partial or complete occlusion of arterioles, and the prevention of blood loss due to the smaller diameters of the arterioles. Whereas the thickest and most readily shed thrombi are produced by the WSR of $5,000 \mathrm{~s}^{-1}$, this may lead to embolization of the distal circulation in markedly stenosed arteries with ruptured atherosclerotic plaques $(3,33)$. This may contribute to intermittent thromboembolic symptoms, which are characteristic of acute coronary syndromes and cerebrovascular disease (33). By contrast, the thicker shell and more densely packed core of the thrombi formed at $5,000 \mathrm{~s}^{-1}$ prevents the penetration of thrombolytic drugs, which makes thrombolysis more difficult.

In conclusion, the results of the present study demonstrated that thrombi characteristics varied with WSRs. With increasing WSRs, an increase in thrombus height was observed, and the total fluorescence intensity and thrombus area exhibited parabolic curve-like changes. The thrombi formed at higher WSRs were thicker and more readily shed, and had a more densely packed core, compared with those formed at lower WSRs, which may cause embolization and inhibit thrombolysis. Therefore, the balance between attachment and detachment of platelets regulated thrombus formation at different WSRs.

\section{Acknowledgements}

This study was supported by grants from the National Natural Science Foundation of China (grant no. 81270594), the National Basic Research Program of China (grant no. 2012CB518000) and the National Natural Science Foundation of China (grant no. 81070414). Dr Xiaofeng Shi was a recipient of a fellowship from the China Postdoctoral Science Foundation (grant no. 2013M541528). The authors would like to thank the International Science Editing Company for manuscript editing.

\section{References}

1. Ruggeri ZM: Platelets in atherothrombosis. Nat Med 8: 1227-1234, 2002

2. Ruggeri ZM, Orje JN, Habermann R, Federici AB and Reininger AJ: Activation-independent platelet adhesion and aggregation under elevated shear stress. Blood 108: 1903-1910, 2006.

3. Kroll MH, Hellums JD, McIntire LV, Schafer AI and Moake JL: Platelets and shear stress. Blood 88: 1525-1541, 1996.

4. Colace TV, Tormoen GW, McCarty OJ and Diamond SL: Microfluidics and coagulation biology. Annu Rev Biomed Eng 15: 283-303, 2013.

5. Feghhi S and Sniadecki NJ: Mechanobiology of platelets: Techniques to study the role of fluid flow and platelet retraction forces at the micro- and nano-scale. Int $\mathrm{J}$ Mol Sci 12:9009-9030, 2011

6. Savage B, Almus-Jacobs F and Ruggeri ZM: Specific synergy of multiple substrate-receptor interactions in platelet thrombus formation under flow. Cell 94: 657-666, 1998.

7. Savage B, Saldivar E and Ruggeri ZM: Initiation of platelet adhesion by arrest onto fibrinogen or translocation on von Willebrand factor. Cell 84: 289-297, 1996.

8. Inoue O, Suzuki-Inoue K and Ozaki Y: Redundant mechanism of platelet adhesion to laminin and collagen under flow: Involvement of von Willebrand factor and glycoprotein Ib-IX-V. J Biol Chem 283: 16279-16282, 2008.

9. Watson SP: Platelet activation by extracellular matrix proteins in haemostasis and thrombosis. Curr Pharm Des 15: 1358-1372, 2009

10. Farndale RW, Sixma JJ, Barnes MJ and de Groot PG: The role of collagen in thrombosis and hemostasis. J Thromb Haemost 2: 561-573, 2004.

11. Heemskerk JW, Sakariassen KS, Zwaginga JJ, Brass LF, Jackson SP and Farndale RW; Biorheology Subcommittee of the SSC of the ISTH: Collagen surfaces to measure thrombus formation under flow: Possibilities for standardization. J Thromb Haemost 9: 856-858, 2011.

12. Affeld K, Goubergrits L, Watanabe N and Kertzscher U: Numerical and experimental evaluation of platelet deposition to collagen coated surface at low shear rates. J Biomech 46: 430-436, 2013.

13. Savage B, Ginsberg MH and Ruggeri ZM: Influence of fibrillar collagen structure on the mechanisms of platelet thrombus formation under flow. Blood 94: 2704-2715, 1999.

14. Neeves KB, Onasoga AA, Hansen RR, Lilly JJ, Venckunaite D, Sumner MB, Irish AT, Brodsky G, Manco-Johnson MJ and Di Paola JA: Sources of variability in platelet accumulation on type 1 fibrillar collagen in microfluidic flow assays. PLoS One 8: e54680, 2013

15. Colace T, Falls E, Zheng XL and Diamond SL: Analysis of morphology of platelet aggregates formed on collagen under laminar blood flow. Ann Biomed Eng 39: 922-929, 2011.

16. Stephens G, He M, Wong C, Jurek M, Luedemann HC, Shapurian G, Munnelly K, Muir C, Conley PB and Phillips DR: Development of a perfusion chamber assay to study in real time the kinetics of thrombosis and the antithrombotic characteristics of antiplatelet drugs. Thromb J 10: 11, 2012.

17. Li M, Hotaling NA, Ku DN and Forest CR: Microfluidic thrombosis under multiple shear rates and antiplatelet therapy doses. PLoS One 9: e82493, 2014.

18. Westein E, van der Meer AD, Kuijpers MJ, Frimat JP, van den Berg A and Heemskerk JW: Atherosclerotic geometries exacerbate pathological thrombus formation poststenosis in a von Willebrand factor-dependent manner. Proc Natl Acad Sci USA 110: 1357-1362, 2013.

19. de Witt SM, Swieringa F, Cavill R, Lamers MM, van Kruchten R, Mastenbroek T, Baaten C, Coort S, Pugh N, Schulz A, et al: Identification of platelet function defects by multi-parameter assessment of thrombus formation. Nat Commun 5: 4257, 2014.

20. StalkerTJ, Traxler EA, Wu J, Wannemacher KM, Cermignano SL, Voronov R, Diamond SL and Brass LF: Hierarchical organization in the hemostatic response and its relationship to the platelet-signaling network. Blood 121: 1875-1885, 2013.

21. Welsh JD, Stalker TJ, Voronov R, Muthard RW, Tomaiuolo M, Diamond SL and Brass LF: A systems approach to hemostasis: 1. The interdependence of thrombus architecture and agonist movements in the gaps between platelets. Blood 124: 1808-1815, 2014. 
22. Stalker TJ, Welsh JD, Tomaiuolo M, Wu J, Colace TV, Diamond SL and Brass LF: A systems approach to hemostasis: 3. Thrombus consolidation regulates intrathrombus solute transport and local thrombin activity. Blood 124: 1824-1831, 2014.

23. Tomaiuolo M, Stalker TJ, Welsh JD, Diamond SL, Sinno T and Brass LF: A systems approach to hemostasis: 2. Computational analysis of molecular transport in the thrombus microenvironment. Blood 124: 1816-1823, 2014.

24. Conant CG, Schwartz MA and Ionescu-Zanetti C: Well plate-coupled microfluidic devices designed for facile image-based cell adhesion and transmigration assays. J Biomol Screen 15: 102-106, 2010.

25. Benoit MR, Conant CG, Ionescu-Zanetti C, Schwartz M and Matin A: New device for high-throughput viability screening of flow biofilms. Appl Environ Microbiol 76: 4136-4142, 2010.

26. Valéra MC, Gratacap MP, Gourdy P, Lenfant F, Cabou C, Toutain CE, Marcellin M, Saint Laurent N, Sié P and Sixou M: Chronic estradiol treatment reduces platelet responses and protects mice from thromboembolism through the hematopoietic estrogen receptor $\alpha$. Blood 120: 1703-1712, 2012.

27. Ono A, Westein E, Hsiao S, Nesbitt WS, Hamilton JR, Schoenwaelder SM and Jackson SP: Identification of a fibrin-independent platelet contractile mechanism regulating primary hemostasis and thrombus growth. Blood 112: 90-99, 2008.

28. Reininger AJ, Heijnen HF, Schumann H, Specht HM, Schramm W and Ruggeri ZM: Mechanism of platelet adhesion to von Willebrand factor and microparticle formation under high shear stress. Blood 107: 3537-3545, 2006.

29. Bao J, Xiao J, Mao Y and Zheng XL: Carboxyl terminus of ADAMTS13 directly inhibits platelet aggregation and ultra large von Willebrand factor string formation under flow in a free-thiol-dependent manner. Arterioscler Thromb Vasc Biol 34: $397-407,2014$
30. Conant CG, Schwartz MA, Beecher JE, Rudoff RC, Ionescu-Zanetti $\mathrm{C}$ and Nevill JT: Well plate microfluidic system for investigation of dynamic platelet behavior under variable shear loads. Biotechnol Bioeng 108: 2978-2987, 2011.

31. Hathcock JJ: Flow effects on coagulation and thrombosis. Arterioscler Thromb Vasc Biol 26: 1729-1737, 2006.

32. Strony J, Beaudoin A, Brands D and Adelman B: Analysis of shear stress and hemodynamic factors in a model of coronary artery stenosis and thrombosis. Am J Physiol 265: H1787-H1796, 1993.

33. Nesbitt WS, Westein E, Tovar-Lopez FJ, Tolouei E, Mitchell A, Fu J, Carberry J, Fouras A and Jackson SP: A shear gradient-dependent platelet aggregation mechanism drives thrombus formation. Nat Med 15: 665-673, 2009.

34. Wong AK: Platelet biology: The role of shear. Expert Rev Hematol 6: 205-212, 2013.

35. Aleman MM, Byrnes JR, Wang JG, Tran R, Lam WA, Di Paola J, Mackman N, Degen JL, Flick MJ and Wolberg A: Factor XIII activity mediates red blood cell retention in venous thrombi. J Clin Invest 124: 3590-3600, 2014.

36. Maxwell MJ, Westein E, Nesbitt WS, Giuliano S, Dopheide SM and Jackson SP: Identification of a 2-stage platelet aggregation process mediating shear-dependent thrombus formation. Blood 109: 566-576, 2007.

37. Huang PY and Hellums JD: Aggregation and disaggregation kinetics of human blood platelets: Part II. Shear-induced platelet aggregation. Biophys J 65: 344-353, 1993.

38. Wootton DM and Ku DN: Fluid mechanics of vascular systems, diseases and thrombosis. Annu Rev Biomed Eng 1: 299-329, 1999.

39. Basmadjian D: Embolization: Critical thrombus height, shear rates and pulsatility. Patency of blood vessels. J Biomed Mater Res 23: 1315-1326, 1989. 